\title{
Unique Continuation for the Kawahara Equation
}

P.N. da SILVA, Departamento de Análise, Rua São Francisco Xavier, 524, Sala 6006, Bloco D, 20550-013 Rio de Janeiro, RJ, Brasil.

Abstract. We establish a unique continuation property for the Kawahara equation. To state such property, we use a Carleman inequality for a linear differential operator related to the Kawahara equation.

\section{Introduction}

We consider the Kawahara equation

$$
u_{t}+u_{x x x}+\eta u_{x x x x x}+u u_{x}=0
$$

where $t \in(-T, T), x \in \Omega, \Omega$ is an open interval in $\mathbb{R}$, and $\eta \neq 0$.

This equation is related to one-dimensional evolution of small amplitude long waves in several problems arising in fluid dynamics. Nonlinear dispersive problems have been object of intensive research (see, for instance, the classical paper of Benjamin-Bona-Mahony [1], Biagioni-Linares [2], Menzala et al. [4], Rosier [5] and the references therein).

This work is concerned with a unique continuation result for the Kawahara equation. Let $L$ be the following operator

$$
L u=u_{t}+u_{x x x}+\eta u_{x x x x x}+u u_{x} .
$$

We assume that $L$ acts on functions defined on some open connected set $\mathcal{Q}$ of $\mathbb{R}^{2}=\mathbb{R}_{x} \times \mathbb{R}_{t}$. L is said to have the unique continuation property (UCP) if every solution $u$ of $L u=0$ which vanishes on some nonempty open set $\mathcal{O}$ of $\mathcal{Q}$ vanishes in the horizontal component of $\mathcal{O}$ in $\mathcal{Q}$, that is, in $\left\{(x, t) \in \mathcal{Q}, \exists x_{1},\left(x_{1}, t\right) \in \mathcal{O}\right\}$.

We establish the UCP for the following operator

$$
L=\frac{\partial}{\partial t}+\frac{\partial^{3}}{\partial x^{3}}+\eta \frac{\partial^{5}}{\partial x^{5}}+r(x, t) \frac{\partial}{\partial x}
$$

and, as a consequence, we establish our main result:

Theorem 1.1. Let $\mathcal{Q}$ the cylinder $\Omega \times(-T, T)$, where $\Omega$ is an open interval in $\mathbb{R}$. Let $L$ be the operator defined by $(1.2)$. Let $u \in L^{2}\left(-T, T ; H^{5}(\Omega)\right) \cap L^{\infty}\left(-T, T ; L_{l o c}^{2}(\Omega)\right)$ be a solution of $L u=0$, that vanishes in some open set $\mathcal{O} \subset \mathcal{Q}$. Then u vanishes at the horizontal component of $\mathcal{O}$.

\footnotetext{
${ }^{1}$ nunes@ime.uerj.br
} 
Kenig [3] pointed out that the UCP obtained by Zhang [9], by means of the scattering theory argument, may not be applied for a Kawahara type equation.

In this paper, we follow from close the work by Saut and Scheurer [6] on UCP, especially the treatment for linear dispersive operators of one space dimension. Our analysis is based on a Carleman type estimate for a linearized equation associated to $(1.1)$.

In Section 2., we give some notations and we present an useful corollary of the Trèves' inequality. In Section 3., we give some useful technical results: we establish a Carleman type estimate for two linear operator related to operator (1.2); we prove, for these operators, that if a solution vanishes in a ball in the $x t$ plane, which pass through the origin, then it also vanishes in a neighborhood of the origin. We also obtain result that provides an uniform control in that neighborhood of the origin. The Section 4. contains our main result and its proof. In Section 5., we apply the UCP result for a Kawahara system in a bounded interval $(0, T)$.

\section{Notation and Trèves' Inequality}

In what follows we are going to use the notation $D_{j}=\frac{\partial}{\partial x_{j}}, 1 \leq j \leq n$, and $D=\left(D_{1}, \ldots, D_{n}\right)$. If $X=\left(X_{1}, \ldots, X_{n}\right)$, let $C[X]$ be the algebra of polynomials in $n$ variables. If $P \in C[X]$ and $P$ has constant coefficients and degree $m$, then we consider the differential operator $P(D)=\sum_{|\alpha| \leq m} a_{\alpha} D^{\alpha}$ of order $m$, where $D^{\alpha}=D_{1}^{\alpha_{1}} \cdots D_{n}^{\alpha_{n}}$ and $|\alpha|=\sum_{j=1}^{n} \alpha_{j}$. By definition, $P^{(\beta)}(X)=\frac{\partial^{|\beta|} P(X)}{\partial x_{1}^{\beta_{1}} \ldots \partial x_{n}^{\beta_{n}}}$ where $\beta$ is given by $\beta=\left(\beta_{1}, \ldots, \beta_{n}\right) \in \mathbb{N}^{n}$.

In this section, we follow from close the arguments of Shang [7].

Theorem 2.1 (Trèves' Inequality). Let $P=P(D)$ be a differential operator of order $m$ with constant coefficients. Then, for any multi-index $\alpha \in \mathbb{N}^{n}, \xi \in \mathbb{R}^{n}$ and $\phi \in C_{0}^{\infty}\left(\mathbb{R}^{n}\right)$ we have the inequality

$$
\frac{2^{|\alpha|}}{\alpha !} \xi^{2 \alpha} \int_{\mathbb{R}^{n}}\left|P^{(\alpha)}(D) \phi\right|^{2} \exp (\psi(y, \xi)) d y \leq C(m, \alpha) \int_{\mathbb{R}^{n}}|P(D) \phi|^{2} \exp (\psi(y, \xi)) d y
$$

where $\psi(y, \xi)=\sum_{i=1}^{n} y_{i}^{2} \xi_{i}^{2}, \xi^{2 \alpha}=\xi_{1}^{2 \alpha_{1}} \ldots \xi_{n}^{2 \alpha_{n}}$, with $\xi=\left(\xi_{1}, \ldots, \xi_{n}\right)$. The constant $C(m, \alpha)$ is given by $C(m, \alpha)=\left\{\begin{array}{ll}\sup _{|r+\alpha| \leq m}\left(\begin{array}{c}r+\alpha \\ \alpha\end{array}\right), & |\alpha| \leq m \\ 0, & |\alpha|>m\end{array}\right.$ where $\alpha, r \in \mathbb{N}^{n}$ and $\alpha !=\alpha_{1} ! \cdots \alpha_{n} !$, if $\alpha=\left(\alpha_{1}, \ldots, \alpha_{n}\right)$.

Corollary 2.1. Let $P=P(D)=P\left(\frac{\partial}{\partial x}, \frac{\partial}{\partial t}\right)$ be a differential operator of order $m$ with constant coefficients. Let $\delta, \tau>0$ be any positive real numbers and $\varphi(x, t)=$ $(x-\delta)^{2}+\delta^{2} t^{2}$. Then the inequality

$$
\frac{2^{2|\alpha|}}{\alpha !} \tau^{|\alpha|} \delta^{2 \alpha_{2}} \int_{\mathbb{R}^{2}}\left|P^{(\alpha)}(D) \phi\right|^{2} \exp (2 \tau \varphi) d y \leq C(m, \alpha) \int_{\mathbb{R}^{2}}|P(D) \phi|^{2} \exp (2 \tau \varphi) d y
$$

holds for all $\phi \in C_{0}^{\infty}\left(\mathbb{R}^{2}\right)$ and $\alpha=\left(\alpha_{1}, \alpha_{2}\right) \in \mathbb{N}^{2}$, such that $|\alpha| \leq m$. 
Proof. We use the Trèves' Inequality for the differential operator given by $Q(D)=P(D+a)=P\left(\frac{\partial}{\partial x}+a, \frac{\partial}{\partial t}\right)$ where $a=2 \tau \delta, \tau>0$. That is, $y=(x, t)$, $\xi=\left(\xi_{1}, \xi_{2}\right)=(\sqrt{2 \tau}, \delta \sqrt{2 \tau})$. Multiply both sides of inequality (2.1) by $\exp \left(2 \tau \delta^{2}\right)$ and by $\exp ( \pm 4 \tau \delta x)$ to obtain

$$
\begin{aligned}
& \frac{2^{|\alpha|}}{\alpha !} \xi^{2 \alpha} \int_{\mathbb{R}^{2}}\left|\exp (2 \tau \delta x) P^{(\alpha)}(D+a) \phi\right|^{2} \exp \left(2 \tau\left(x^{2}-2 \delta x+\delta^{2}+\delta^{2} t^{2}\right)\right) d x d t \\
& \leq C(m, \alpha) \int_{\mathbb{R}^{2}}|\exp (2 \tau \delta x) P(D+a) \phi|^{2} \exp \left(2 \tau\left((x-\delta)^{2}+\delta^{2} t^{2}\right)\right) d x d t
\end{aligned}
$$

Choose $\phi=\widetilde{\phi} \exp (-2 \tau \delta x)$ where $\widetilde{\phi} \in C_{0}^{\infty}\left(\mathbb{R}^{2}\right)$. The proof is complete if we note that $P(D+a)[\widetilde{\phi} \exp (-2 \tau \delta x)]=\exp (-2 \tau \delta x) P(D) \widetilde{\phi}$ and $P^{(\alpha)}(D+a)[\widetilde{\phi} \exp (-2 \tau \delta x)]=$ $\exp (-2 \tau \delta x) P^{(\alpha)}(D) \widetilde{\phi}$.

\section{Technical Results}

In the proof of our main result (Theorem 1.1) we use the following result.

Theorem 3.1. Let $\mathcal{O}$ be the cylinder $\Omega \times(-T, T), \quad \Omega$ an open interval in $\mathbb{R}$. Let $L$ be the operator defined at (1.3). Assume that $r \in L^{\infty}\left(-T, T ; L_{\text {loc }}^{2}(\Omega)\right)$. Let $u \in L^{2}\left(-T, T ; H^{5}(\Omega)\right)$ be a solution of $L u=0$, that vanishes in some open subset $\mathcal{O}_{1} \subset \mathcal{O}$. Then $u$ vanishes on the horizontal component of $\mathcal{O}_{1}$.

In the next subsections, we deduce results that will be useful in the proof of Theorem 3.1. In Subsection 3.1., we deduce a Carleman estimate, in Subsection 3.2., we obtain a UCP for a solution that vanishes in a ball in the $x t$ plane, which pass through the origin and prove Theorem 3.1.

\subsection{Carleman Estimate}

Now, we deduce a Carleman estimate.

Proposition 3.1. Let $\Omega$ be an open interval in $\mathbb{R}$ that contains the origin and $\mathcal{O}=\Omega \times(-T, T), 0<T \leq \infty$. Consider the differential operator

$$
L_{ \pm}=\frac{\partial}{\partial t} \pm \frac{\partial^{3}}{\partial x^{3}} \pm \eta \frac{\partial^{5}}{\partial x^{5}} \pm r(x, t) \frac{\partial}{\partial x},
$$

where $r \in L^{\infty}\left(-T, T, L^{2}(\Omega)\right)$. Then, the following inequality

$$
\begin{aligned}
& \tau \int_{\mathcal{O}}\left|\phi_{x x x x}\right|^{2} \exp (2 \tau \varphi) d x d t+\tau^{2} \int_{\mathcal{O}}\left|\phi_{x x x}\right|^{2} \exp (2 \tau \varphi) d x d t+\tau^{3} \int_{\mathcal{O}}\left|\phi_{x x}\right|^{2} \exp (2 \tau \varphi) d x d t \\
& +\tau^{4} \int_{\mathcal{O}}\left|\phi_{x}\right|^{2} \exp (2 \tau \varphi) d x d t+\tau^{5} \int_{\mathcal{O}}|\phi|^{2} \exp (2 \tau \varphi) d x d t \leq \frac{5}{\eta^{2}} \int_{\mathcal{O}}\left|L_{ \pm} \phi\right|^{2} \exp (2 \tau \varphi) d x d t
\end{aligned}
$$


holds for all $\phi \in C_{0}^{\infty}(\mathcal{O})$, where $\varphi(x, t)=(x-\delta)^{2}+\delta^{2} t^{2}$ with $0<\delta<1, \quad \tau>0$ such that

$$
\tau>\max \left\{C\|r\|_{L^{\infty}\left(-T, T, L^{2}(\Omega)\right)}^{2}, 4 C(|\Omega|+1)^{2}\|r\|_{L^{\infty}\left(-T, T, L^{2}(\Omega)\right)}^{2}, 1, \frac{1}{\eta^{2}},\right\}
$$

and $C$ is the embedding constant of $H^{1}(\Omega)$ into $L^{\infty}(\Omega)$.

Proof. We use Trèves' inequality for the operators $P_{ \pm}=\frac{\partial}{\partial t} \pm \eta \frac{\partial^{5}}{\partial x^{5}}$. With the notation of Section 2., we have $P_{ \pm}\left(\xi_{1}, \xi_{2}\right)=\xi_{2} \pm \eta \xi_{1}^{5}$. For $\alpha=(k, 0)$ and $r=\left(r_{1}, r_{2}\right)$, with $k, r_{1}, r_{2} \in \mathbb{N}$, we have $\left(\begin{array}{c}r+\alpha \\ \alpha\end{array}\right)=\left(\begin{array}{c}\left(r_{1}+k, r_{2}\right) \\ (k, 0)\end{array}\right)=\frac{\left(r_{1}+k\right) ! r_{2} !}{r_{1} ! r_{2} ! k !}=\frac{\left(r_{1}+k\right) !}{r_{1} ! k !}$. Thus the coefficients $C(5, \alpha)$ for $\alpha=(1,0),(2,0), \ldots,(5,0)$ are equal to $5,10,10,5$ and 1 , respectively.

From the estimates obtained by using inequality $(2.2)$ for $\alpha=(1,0),(2,0), \ldots$, $(5,0)$, we deduce that

$$
\begin{aligned}
& \tau \int_{\mathcal{O}}\left|\phi_{x x x x}\right|^{2} \exp (2 \tau \varphi) d x d t+\tau^{2} \int_{\mathcal{O}}\left|\phi_{x x x}\right|^{2} \exp (2 \tau \varphi) d x d t+\tau^{3} \int_{\mathcal{O}}\left|\phi_{x x}\right|^{2} \exp (2 \tau \varphi) d x d t \\
& +\tau^{4} \int_{\mathcal{O}}\left|\phi_{x}\right|^{2} \exp (2 \tau \varphi) d x d t+\tau^{5} \int_{\mathcal{O}}|\phi|^{2} \exp (2 \tau \varphi) d x d t \\
& \leq \frac{3}{20 \eta^{2}} \int_{\mathcal{O}}\left|\phi_{t} \pm \eta \phi_{x x x x x}\right|^{2} \exp (2 \tau \varphi) d x d t .
\end{aligned}
$$

Since

$$
\begin{aligned}
\int_{\Omega}\left|r(x, t) \phi_{x}\right|^{2} \exp (2 \tau \varphi) d x d t \leq\|r(\cdot, t)\|_{L^{2}(\Omega)}^{2}\left\|\phi_{x} \exp (\tau \varphi)\right\|_{L^{\infty}}^{2} \\
\leq C\|r(\cdot, t)\|_{L^{2}(\Omega)}^{2}\left\|\phi_{x} \exp (\tau \varphi)\right\|_{H^{1}(\Omega)}^{2} \\
\leq C\|r(\cdot, t)\|_{L^{2}(\Omega)}^{2}\left[\left\|\phi_{x x} \exp (\tau \varphi)\right\|_{L^{2}(\Omega)}^{2}+\tau^{2} \sup _{x \in \Omega}\left|4(x-\delta)^{2}\right|\left\|\phi_{x} \exp (\tau \varphi)\right\|_{L^{2}(\Omega)}^{2}\right. \\
\left.\quad+\left\|\phi_{x} \exp (\tau \varphi)\right\|_{L^{2}(\Omega)}^{2}\right],
\end{aligned}
$$

if $\tau$ satisfies (3.2), we have

$$
\int_{\Omega}\left|r(x, t) \phi_{x}\right|^{2} \exp (2 \tau \varphi) d x d t \leq \tau^{3} \eta^{2}\left\|\phi_{x x} \exp (\tau \varphi)\right\|_{L^{2}(\Omega)}^{2}+2 \tau^{4} \eta^{2}\left\|\phi_{x} \exp (\tau \varphi)\right\|_{L^{2}(\Omega)}^{2} .
$$

Thus, by (3.3) and (3.4),

$$
\int_{\mathcal{O}}\left[\left|\phi_{x x x}\right|^{2}+\left|r(x, t) \phi_{x}\right|^{2}\right] \exp (2 \tau \varphi) d x d t \leq \frac{3}{10} \int_{\mathcal{O}} \phi_{t} \pm\left.\eta \phi_{x x x x x}\right|^{2} \exp (2 \tau \varphi) d x d t
$$

Since

$$
\left|\phi_{t} \pm \eta \phi_{x x x x x}\right|^{2}=\left|L_{ \pm} \phi \mp \phi_{x x x} \mp r(x, t) \phi_{x}\right|^{2} \leq 3\left|L_{ \pm} \phi\right|^{2}+3\left|\phi_{x x x}\right|^{2}+3\left|r(x, t) \phi_{x}\right|^{2},
$$


the right hand side of (3.5) is bounded by

$$
\frac{9}{10} \int_{\mathcal{O}}\left|L_{ \pm} \phi\right|^{2} \exp (2 \tau \varphi) d x d t+\frac{9}{10} \int_{\mathcal{O}}\left[\left|\phi_{x x x}\right|^{2}+\left|r(x, t) \phi_{x}\right|^{2}\right] \exp (2 \tau \varphi) d x d t
$$

Now, from (3.5) and (3.7), we may deduce that

$$
\int_{\mathcal{O}}\left[\left|\phi_{x x x}\right|^{2}+\left|r(x, t) \phi_{x}\right|^{2}\right] \exp (2 \tau \varphi) d x d t \leq 9 \int_{\mathcal{O}}\left|L_{ \pm} \phi\right|^{2} \exp (2 \tau \varphi) d x d t .
$$

Returning to inequality (3.3), using the above estimate and (3.6), we complete the proof.

Corollary 3.1. Let $T>0$. Under the assumptions of Proposition 3.1, the inequality (3.1) holds if we replace $\phi(x, t)$ by a function $v(x, t)$ such that $v \in L^{2}\left(-T, T, H^{5}(\Omega)\right)$ with $v_{t} \in L^{2}\left(-T, T, L_{l o c}^{2}(\Omega)\right)$ and such that the support of $v$ is a compact subset of $\mathcal{O}$.

\section{2. $\quad$ Proof of Theorem 3.1}

Now we prove Theorem 3.1 following the steps indicated by Saut and Scheurer [6]. We make several reductions in order to apply the Carleman estimate (Proposition 3.1). The steps consist in:

1. Change of Variable We consider a noncharacteristic curve $\chi(x, t)=0$ that satisfies $\chi(0,0)=0$ and $\frac{\partial \chi}{\partial x}(0,0) \neq 0$. The equation $\chi(x, t)=0$ can therefore locally be written as $x=\psi(t)$. By the change of variable $(x, t) \mapsto(x-\psi(t), t)$, one can suppose that $\psi \equiv 0$.

2. Holmgren Transform We are then reduced to prove the uniqueness in the Cauchy problem across the curve $x=0$ and in a neighborhood of the origin. This curve is convexified by the Holmgren transform $(x, t) \mapsto\left(x+t^{2}, t\right)$

3. Carleman Estimate We shall apply the Carleman estimate (Proposition 3.1) for the resulting operator.

4. Connectiveness The final result follows by connectiveness.

In Lemmas 3.1 and 3.2, we fulfill steps 1 and 2, in order to apply the Carleman estimate and obtain the UCP for a solution that vanishes in a ball in the $x t$ plane, which pass through the origin.

Lemma 3.1. Let $\Omega$ be an open interval of $\mathbb{R}$ which contains the origin and let $\mathcal{O}=\Omega \times(-T, T)$, with $0<T \leq \infty$. Consider the differential operator (1.3) where $r \in L^{\infty}\left(-T, T ; L_{\text {loc }}^{2}(\Omega)\right)$. Let $u=u(x, t)$ be a solution of $L u=0$ in $\mathcal{O}$ such that $u \in L^{2}\left(-T, T, H^{5}(\Omega)\right)$. Let $\gamma$ be a circumference passing through the origin. Suppose that $u \equiv 0$ in the interior of the circle (with boundary $\gamma$ ) centered in $(\alpha, \beta)$, with $\alpha<0$, and contained in $\mathcal{O}$. Then, there exists a neighborhood $\mathcal{O}_{1}$ of the origin (in the $x$ t plane) such that $u \equiv 0$ in $\mathcal{O}_{1}$. 
Proof. The curve $\gamma$ is given by $(x-\alpha)^{2}+(t-\beta)^{2}=\alpha^{2}+\beta^{2} \neq 0, \alpha<0$. Let us define

$$
g(t)=\alpha+\sqrt{\alpha^{2}+\beta^{2}-(t-\beta)^{2}}, \quad t \in I=\left(\beta-\sqrt{\alpha^{2}+\beta^{2}}, \beta+\sqrt{\alpha^{2}+\beta^{2}}\right) .
$$

Since $u \equiv 0$ in the interior of the circle centered at $(\alpha, \beta)$ with radius $\sqrt{\alpha^{2}+\beta^{2}}$, then $u \equiv 0$ in the region $\{(x, t), \alpha<x<g(t), t \in I\}$.

Let us consider the change of variables: $(x, t) \rightarrow\left(x-g(t)+t^{2}, t\right)=(w, t)$. Let $q(w, t)=u(x, t)$. Since $L u=0$, we have

$$
\begin{aligned}
q_{t} & +q_{w w w}+\eta q_{w w w w w}+\left(2 t-g^{\prime}(t)+r\left(w+g(t)-t^{2}, t\right)\right) q_{w} \\
& =u_{t}+u_{x x x}+\eta u_{x x x x x}+r(x, t) u_{x}=0
\end{aligned}
$$

Thus, $\widehat{L} q=0$ where $\widehat{L}$ is given by $\widehat{L}=\frac{\partial}{\partial t}+\frac{\partial^{3}}{\partial w^{3}}+\eta \frac{\partial^{5}}{\partial w^{5}}+\widehat{r}(w, t) \frac{\partial}{\partial w}$, with $\widehat{r}(w, t)=2 t-g^{\prime}(t)+r\left(w-t^{2}+g(t), t\right)$. We also have $q \equiv 0$ in the region $\mathcal{R}=$ $\left\{(w, t), t^{2}-\sqrt{\alpha^{2}+\beta^{2}-(t-\beta)^{2}}<w<t^{2}\right\}$.

Let $0<\delta<1$ and $B_{\delta}=\left\{(w, t) \in \mathbb{R}^{2}, w^{2}+t^{2}<\delta^{2}\right\}$. Let $h \in C_{0}^{\infty}\left(B_{\delta}\right)$ such that $h \equiv 1$ in a neighborhood $\mathcal{O}_{1}$ of the origin; let $v=v(x, t)=h q$. It follows that $v \in L^{2}\left(-T, T, H^{5}(\mathcal{O})\right)$ and $v$ has compact support in $B_{\delta}$.

If $(w, t) \in B_{\delta} \cap \operatorname{supp} v$, then $(w-\delta)^{2}+\delta^{2} t^{2}<\delta^{2}$. Thus, $\operatorname{supp}(v) \subset\{(w, t),(w-$ $\left.\delta)^{2}+\delta^{2} t^{2} \leq \delta^{2}\right\} \equiv\{(w, t), \varphi(w, t) \leq \varphi(0,0)\}$, where $\varphi(w, t)=(w-\delta)^{2}+\delta^{2} t^{2}$. Assume $\tau$ satisfies (3.2). Since $h=1$ in a neighborhood $\mathcal{O}_{1}$ of the origin, it follows by Corollary 3.1 that

$$
\tau^{5} \eta^{2} \int_{B_{\delta}}|v|^{2} \exp (2 \tau \varphi) d w d t \leq 5 \int_{B_{\delta}-\mathcal{O}_{1}}|\widehat{L} v|^{2} \exp (2 \tau \varphi) d w d t
$$

Consider the sets $A=\left(\left[\overline{B_{\delta}} \backslash \mathcal{O}_{1}\right] \cap\left\{(w, t), t^{2} \leq w\right\}\right)$ and $B=\{(w, t), \varphi(w, t)=$ $\left.\varphi(0,0)=\delta^{2}\right\}$. Note that the intersection of $A$ and $B$ is the empty set. Therefore, there exists $\varepsilon>0,\left(\varepsilon<\delta^{2}\right)$ such that $\varphi(w, t) \leq \delta^{2}-\varepsilon$ for all $(w, t) \in A$. Now, let $\mathcal{O}_{2}$ be a neighborhood of the origin such that $\varphi(w, t)>\delta^{2}-\varepsilon$ in $\mathcal{O}_{2}$. Using (3.8), we deduce that

$$
K e^{2 \tau\left(\delta^{2}-\varepsilon\right)} \int_{\mathcal{O}_{2}}|v|^{2} d w d t \leq \int_{B_{\delta}-\mathcal{O}_{1}}|\widehat{L} v|^{2} e^{2 \tau \varphi} d w d t \leq e^{2 \tau\left(\delta^{2}-\varepsilon\right)} \int_{B_{\delta}-\mathcal{O}_{1}}|\widehat{L} v|^{2} d w d t
$$

where $K=\frac{\tau^{4}}{5}$. That is

$$
\int_{\mathcal{O}_{2}}|v|^{2} d w d t \leq \frac{5}{\tau^{4}} \int_{B_{\delta}-\mathcal{O}_{1}}|\widehat{L} v|^{2} d w d t
$$

If we take $\tau \rightarrow \infty$ in (3.9), we conclude that $v \equiv 0$ in $\mathcal{O}_{2}$. Since $q \equiv v$ in $\mathcal{O}_{2} \subset \mathcal{O}_{1}$, then the proof of Lemma 3.1 is complete.

Lemma 3.2. Let $\Omega$ be an open interval of $\mathbb{R}$ which contains the origin and let $\mathcal{O}=\Omega \times(-T, T)$, with $0<T \leq \infty$. Consider the differential operator (1.3) where $r \in L^{\infty}\left(-T, T ; L_{l o c}^{2}(\Omega)\right)$. Let $u=u(x, t)$ be a solution of $L u=0$ in $\mathcal{O}$ such that $u \in L^{2}\left(-T, T, H^{5}(\Omega)\right)$. Let $\gamma$ be a circumference passing through the origin. Suppose that $u \equiv 0$ in the interior of the circle (with boundary $\gamma$ ) centered in $(\alpha, \beta)$, with $\alpha>0$, contained in $\mathcal{O}$. Then, there exists a neighborhood $\mathcal{O}_{2}$ of the origin (in the xt plane) such that $u \equiv 0$ in $\mathcal{O}_{2}$. 
Proof. The curve $\gamma$ is given by $(x-\alpha)^{2}+(t-\beta)^{2}=\alpha^{2}+\beta^{2} \neq 0, \alpha>0$. Consider the change of variable $(x, t) \rightarrow(-w, t)$.

In the new variables, the operator $L$ is written as

$$
L=\frac{\partial}{\partial t}-\frac{\partial^{3}}{\partial w^{3}}-\eta \frac{\partial^{5}}{\partial w^{5}}-\widetilde{r}(w, t) \frac{\partial}{\partial w},
$$

where $\widetilde{r}(w, t)=r(-w, t)$.

Let $v(w, t)=u(x, t)$. Then $L v=0(L$ given by $(3.10))$ and $v \equiv 0$ in the interior of the circle (with boundary $\gamma$ ), center $(-\alpha, \beta)$ with $-\alpha<0$, which is a subset of $\widetilde{\mathcal{O}}$.

Arguing as in the proof of Lemma 3.1, we can prove Lemma 3.2.

At the connectiveness step of the proof of Theorem 3.1, we should guarantee that given a ball of radius $r_{1}$, we can choose as the neighborhood $\mathcal{O}_{3}$ of the origin the ball $B\left((0,0), r_{1}-|\beta|\right)$, where $r_{1}^{2}=\alpha^{2}+\beta^{2}$. In the next lemma, we obtain the desired result if $r_{1}$ is sufficiently small.

Lemma 3.3. Let $\Omega$ be an open interval of $\mathbb{R}$ which contains the origin and let $\mathcal{O}=\Omega \times(-T, T)$, with $0<T \leq \infty$. Consider the differential operator (1.3) where $r \in L^{\infty}\left(-T, T ; L_{l o c}^{2}(\Omega)\right)$. Let $u=u(x, t)$ be a solution of $L u=0$ in $\mathcal{O}$ such that $u \in L^{2}\left(-T, T, H^{5}(\Omega)\right)$. Let $\gamma$ be a circumference passing through the origin. Suppose that $u \equiv 0$ in the interior of the circle (with boundary $\gamma$ ) centered in $(\alpha, \beta)$, with $\alpha \neq 0$, contained in $\mathcal{O}$ and $r_{1}^{2}=\alpha^{2}+\beta^{2}<\widetilde{r}^{2}$ where $\widetilde{r}$ is given by

$$
\sqrt{7+2 \sqrt{5}} \widetilde{r}=\min \left\{r^{*}(\delta), \frac{\delta(2-\sqrt{\delta(4-\delta)})}{2}, 1\right\}
$$

with $r^{*}(\delta)$ defined on (3.12). Then $u \equiv 0$ in $B\left((0,0), r_{1}-|\beta|\right) \cap \mathcal{O}$.

Proof. We argue as in proofs of Lemmas 3.1 and 3.2. For sake of simplicity, we assume that $\alpha<0$. Let $\widehat{r}=r_{1}-|\beta|$. After performing the change of variables presented at Lemma 3.1 , it is possible to show that the $B((0,0), \widehat{r})$ in the original $x t$ plane is mapped into a subset of $B\left((0,0), r_{b}\right) \cap\{|t|<\widehat{r}\}$ of the $x t$ plane considered after the Holmgren transform step, where $r_{b}=\sqrt{7+2 \sqrt{5}} \hat{r}$.

We want to choose $r_{b}, 0<r_{b}<\delta<1$, that guarantees the existence of a point $\left(x_{b}, t_{b}\right) \in\left\{(x, t) ; \varphi(x, t)=\varphi\left(r_{b}, 0\right)\right\} \cap\left\{x=t^{2}\right\}$ such that $\left\|\left(x_{b}, t_{b}\right)\right\|<\delta$.

First we note that $\left\{(x, t) ; \varphi(x, t)=\varphi\left(r_{b}, 0\right)\right\} \cap\left\{x=t^{2}\right\} \neq \emptyset$ if there exists a real root of the equation

$$
x^{2}+x \delta(\delta-2)+\delta^{2}-\left(r_{b}-\delta\right)^{2}=0 .
$$

That is, we must have $r_{b}<\frac{\delta(2-\sqrt{\delta(4-\delta)})}{2}$. For $0<\delta<2$, let $s_{1}$ be the real positive root of equation (3.11) given by $s_{1}=\frac{-\delta(\delta-2)-\sqrt{\Delta}}{2}$. Let $x_{b}=s_{1}$ and $t_{b}^{2}=x_{b}$. It remains to show that we have $\left\|\left(x_{b}, t_{b}\right)\right\|<\delta$. Let us define $x_{\delta}=\frac{-1+\sqrt{1+4 \delta^{2}}}{2}$. Observe that $\left(x_{\delta}, \sqrt{x_{\delta}}\right) \in B((0,0), \delta) \cap\left\{x=t^{2}\right\}$. If we have $0<x_{b}<x_{\delta}$, we obtain 
$\left\|\left(x_{b}, t_{b}\right)\right\|^{2}<\delta^{2}$. Indeed, first we note that $\lim _{r_{b} \rightarrow 0} x_{b}=\frac{-\delta(\delta-2)-|\delta(\delta-2)|}{2}=0$. Therefore, for $0<\delta<1$,

there exists $r^{*}>0$ such that for $0<r_{b}<r^{*}$, we have $x_{b}<x_{\delta}$

Given $0<\delta<1$, we argue as in the proof of Lemma 3.1. At the end of the proof of Lemma 3.1, we take $\mathcal{O}_{1}=B\left((0,0), r_{2}\right) \cap\left\{\beta-r_{1}<t<\beta+r_{1}\right\}$ where $r_{2}=\left\|\left(x_{b}, t_{b}\right)\right\|, x_{b}=s_{1}, t_{b}=\sqrt{x_{b}}$. By construction, we can conclude that $u$ vanishes in the interior of the ball $B((0,0), r)$ for any $r<r_{1}-|\beta|$. Thus, it also vanishes in $B\left((0,0), r_{1}-|\beta|\right)$. The case $\alpha>0$ is analogous.

\section{Proof of Theorem 3.1}

We denote the horizontal component of $\mathcal{O}_{1}$ by $\mathcal{H}_{1}$. Note that $\mathcal{O}_{1} \subset \mathcal{H}_{1}$. Let

$$
\Lambda=\left\{(x, t) \in \mathcal{H}_{1} \quad \text { such that } \quad u \equiv 0 \quad \text { in a neighborhood of }(x, t)\right\} .
$$

We want to show that $\Lambda=\mathcal{H}_{1}$. Suppose they are not equal. That is, there exists $Q=(\bar{x}, \bar{t}) \in \mathcal{H}_{1} \backslash \Lambda$. Since $Q \in \mathcal{H}_{1}$, there exists $x_{1} \in \mathcal{O}_{1}$ such that $P=\left(x_{1}, \bar{t}\right) \in \mathcal{O}_{1}$. Let $\Gamma$ be the curve parameterized by $f:[0,1] \rightarrow \mathcal{H}_{1}$, with $f(s)=\left(x_{1}+s\left(\bar{x}-x_{1}\right), \bar{t}\right)$. We have $f(0)=P$ and $f(1)=Q$. Let $r_{0}>0$, $r_{0}<\operatorname{dist}\left(\Gamma, \partial \mathcal{H}_{1}\right)$ (where $\partial \mathcal{H}_{1}$ denotes the boundary of $\left.\mathcal{H}_{1}\right)$ such that $B\left(P, r_{0}\right) \subset$ $\Lambda$. Let $r_{1}<\min \left\{\frac{r_{0}}{4}, \widetilde{r}(1 / 2)\right\}, \widetilde{r}$ given by Lemma 3.3. Consider the set $\Lambda_{1}=$ $\left\{(x, t) \in \Lambda\right.$ such that $u \equiv 0$ in $\left.B\left((x, t), r_{1}\right) \cap \mathcal{H}_{1}\right\}$. Let $s_{0}=\sup \{0 \leq s \leq$ 1 , such that $f(\tau) \in \Lambda_{1}$ whenever $\left.0 \leq \tau \leq s\right\}$. We claim that $s_{0}=1$. In fact, first note that $\overline{B\left(f(s), 2 r_{1}\right)} \subset \mathcal{H}_{1}$ for all $s \in[0,1]$. We also have $f\left(s_{0}\right) \in \Lambda_{1}$. Finally, let us suppose that $s_{0}<1$. Let $\widetilde{\varepsilon}>0$ be such that

$$
\widetilde{\varepsilon}<\min \left\{1-s_{0}, \frac{r_{1}}{2}\right\} .
$$

Consider $W_{\varepsilon}=f\left(s_{0}+\varepsilon\right)$ for some $\varepsilon$ such that $0<\varepsilon<\widetilde{\varepsilon}$. Now, we argue that $u$ vanishes in $B\left(W_{\varepsilon}, r_{1}\right)$ for all $0<\varepsilon<\widetilde{\varepsilon}$. This contradicts the definition of $s_{0}$.

Let $F=\left\{w \in \mathcal{H}_{1},\left\|w-f\left(s_{0}\right)\right\|=r_{1}\right\} \cap \overline{B\left(f\left(s_{0}+\varepsilon\right), r_{1}\right)}$. For each element $w_{0}=\left(x_{0}, t_{0}\right)$ of $F$, by Lemma 3.3 , we have $u \equiv 0$ in $B\left(w_{0}, \delta_{2}\left(w_{0}\right)\right)$ for $\delta_{2}\left(w_{0}\right)=$ $r_{1}-\left|t_{0}\right|$. The family $\left\{B\left(w_{0}, \delta_{2}\left(w_{0}\right)\right)\right\}_{w_{0} \in F}$ is an open cover of the region $\overline{B\left(W_{\varepsilon}, r_{1}\right)} \backslash$ $B\left(f\left(s_{0}\right), r_{1}\right)$ except by the points $w_{r_{1}}^{ \pm}=\left(x_{1}+\left(s_{0}+\varepsilon\right)\left(\bar{x}-x_{1}\right), \pm r_{1}\right)$

For each $n \in \mathbb{N}$, we can extract a finite cover from the family $\left\{B\left(w_{0}, \delta_{2}\left(w_{0}\right)\right)\right\}_{w_{0} \in F}$ for the compact set

$$
B_{n}=\overline{B\left(W_{\varepsilon}, r_{1}\right)} \backslash\left(B\left(f\left(s_{0}\right), r_{1}\right) \cup B\left(w_{r_{1}}^{+}, \frac{1}{n}\right) \cup B\left(w_{r_{1}}^{-}, \frac{1}{n}\right)\right) .
$$

We also have $u \equiv 0$ in each $B_{n}$. On the other hand, since $B\left(W_{\varepsilon}, r_{1}\right) \backslash B\left(f\left(s_{0}\right), r_{1}\right)=$ $\cup_{n} B_{n}$ we obtain that $u$ vanishes in $B\left(W_{\varepsilon}, r_{1}\right)$ for all $0<\varepsilon<\widetilde{\varepsilon}$. Thus, $\mathcal{H}_{1}=\Lambda$ and the proof of Theorem 3.1 is complete. 


\section{Unique Continuation Property for the Kawa- hara Equation}

In this section, we prove our main result

Theorem 4.1 (UCP). Let $\mathcal{Q}$ be the cylinder $\Omega \times(-T, T)$, where $\Omega$ is an open interval in $\mathbb{R}$ which contains the origin. Consider $L$ the operator defined by (1.2):

$$
\frac{\partial u}{\partial t}+\frac{\partial^{3} u}{\partial x^{3}}+\eta \frac{\partial^{5}}{\partial x^{5}}+u \frac{\partial u}{\partial x}=0
$$

If $u \in L^{\infty}\left(-T, T ; H^{5}(\Omega)\right) \cap L^{\infty}\left(-T, T ; L_{l o c}^{2}(\Omega)\right)$ is a solution of $L u=0$, that vanishes in some open set $\mathcal{O}_{1} \subset \mathcal{Q}$, then $u$ vanishes at the horizontal component of $\mathcal{O}_{1}$.

Proof. Consider the differential operator (1.3), where $r(x, t)=u(x, t)$. We have $r \in L^{\infty}\left(-T, T, L_{l o c}^{2}(\Omega)\right)$. Since $L u=0$ and considering that $u$ that vanishes in the open set $\mathcal{O}_{1} \subset \mathcal{Q}$; by Theorem 3.1 we obtain that $u$ vanishes at the horizontal component of $\mathcal{O}_{1}$.

Remark. Consider the operator

$$
L=\frac{\partial}{\partial t}+\eta \frac{\partial^{2 k+1}}{\partial x^{2 k+1}}+R\left(x, t, \frac{\partial}{\partial x}\right)+N\left(x, t, \frac{\partial}{\partial x}\right), \quad \eta \neq 0
$$

where $R\left(x, t, \frac{\partial}{\partial x}\right)$ is a differential operator of order $\leq 2 k$ given by $R\left(x, t, \frac{\partial}{\partial x}\right)=$ $\sum_{j \leq 2 k} r_{j} \frac{\partial^{j}}{\partial x^{j}}$, with $r_{j} \in L^{\infty}\left(-T, T ; L_{l o c}^{2}(\Omega)\right)$ and $N\left(x, t, \frac{\partial}{\partial x}\right) u=\frac{\partial u^{p+1}}{\partial x}$, where $p \geq 1$ is an integer.

We point out that arguing as in the case of the Kawahara equation, it is possible to get the following result

Theorem 4.2. Let $\mathcal{Q}$ be the cylinder $\Omega \times(-T, T), \Omega$ an open interval in $\mathbb{R}$ which contains the origin. Consider the operator L given by (4.1). Assume: $r_{j} \in L_{\text {loc }}^{\infty}(\mathcal{Q})$. If $u \in L^{2}\left(-T, T ; H^{2 k+1}(\Omega)\right) \cap L^{\infty}\left(-T, T ; L_{l o c}^{2 p}(\Omega)\right)$ is a solution of $L u=0$ that vanishes in some open set $\mathcal{O} \subset \mathcal{Q}$, then $u$ vanishes at the horizontal component of $\mathcal{O}$.

\section{Kawahara System}

We consider the Kawahara system in a bounded interval $(0, T)$

$$
\begin{cases}u_{t}+u_{x x x}+\eta u_{x x x x x}+\lambda u u_{x}=0, & \text { in }(0, L) \times(0, T), \\ u(0, t)=u(L, t)=0, & \text { for all } t \in(0, T), \\ u_{x}(0, t)=u_{x}(L, t)=0, & \text { for all } t \in(0, T), \\ u_{x x}(L, t)=0, & \text { for all } t \in(0, T),\end{cases}
$$

where $\eta<0, \lambda>0$ and $T>0$. 
In [8], Vasconcellos studied this system and pointed out UCP results for the Kawahara equation are important and useful. Here, we consider, as described by Vasconcellos [8], a solution of the system (5.1) which vanishes on $\omega \times(0, T)$ where $\omega$ is a non-empty subset of $(0, L)$. We establish the following UCP for the system (5.1).

Theorem 5.1. Let $\mathcal{Q}$ the set $(0, L) \times(0, T)$ and $L$ be the operator

$$
L u=\frac{\partial u}{\partial t}+\frac{\partial^{3} u}{\partial x^{3}}+\eta \frac{\partial^{5} u}{\partial x^{5}}+\lambda u \frac{\partial u}{\partial x}=0 .
$$

Assume that $u \in L^{2}\left(0, T ; H^{5}(0, L)\right) \cap L^{2}\left(0, T ; H_{0}^{2}(0, L)\right) \cap L^{\infty}\left(0, T ; L_{\text {loc }}^{2}(0, L)\right)$ is a solution of $L u=0$, that vanishes in the open set $\mathcal{O}_{1}=\omega \times(0, T)$, where $\omega$ is an open, non-empty subset of $(0, L)$. Then $u$ vanishes at $(0, L) \times(0, T)$.

Proof. Let $v(x, t)=u\left(\frac{x+L}{\sqrt[3]{2}}, \frac{t+T}{2}\right), \Omega=(-L,(\sqrt[3]{2}-1) L)$ and $\mathcal{Q}=\Omega \times(-T, T)$. We have that $v \in L^{2}\left(-T, T ; H^{5}(\Omega)\right) \cap L^{2}\left(-T, T ; H_{0}^{2}(\Omega)\right) \cap L^{\infty}\left(-T, T ; L^{2}(\Omega)\right)$ and $v$ satisfies $\widetilde{L} v=0$ where

$$
\widetilde{L} v=v_{t}+v_{x x x}+\widetilde{\eta} v_{x x x x x}+\widetilde{\lambda} v v_{x}
$$

with $\widetilde{\eta}=\frac{\eta}{\sqrt[3]{4}}$ and $\widetilde{\lambda}=\sqrt[3]{4} \lambda$. We also have that $v \equiv 0$ on the subset $\mathcal{O}_{1}=I(\omega) \times$ $(-T, T)$ where $I(\omega)=\left\{I(x)=\frac{x+L}{\sqrt[3]{2}}, x \in \omega\right\}$.

Using the regularity of $u$ and the fact $L u=0$, we have that $r \in L^{\infty}\left(-T, T, L^{2}(\Omega)\right)$. Consider the differential operator (1.3), where $\eta=\widetilde{\eta}$ and $r(x, t)=\widetilde{\lambda} v$. Since $\widehat{L} v=0$ and $v$ vanishes in the open set $\mathcal{O}_{1} \subset \mathcal{Q}$, by Theorem 3.1, we obtain $v$ vanishes at the horizontal component of each connected component of $\mathcal{O}_{1}$. That is $v$ vanishes at $\mathcal{Q}$. Thus $u$ vanishes at $(0, L) \times(0, T)$.

\section{Acknowledgments}

The author thanks C. F. Vasconcellos for his suggestions and comments.

\section{References}

[1] T.B. Benjamin, J.L. Bona, J.J. Mahony, Model equations for long waves in nonlinear dispersive systems, Philos. Trans. Roy. Soc. London, Ser. A 272 (1972), 47-78.

[2] H.A. Biagioni, F. Linares, On the Benney-Lin and Kawahara equations, J. Math. Anal. Appl., 211 (1997), 131-152.

[3] C.E. Kenig, "Some Recent Quantitative Unique Continuation Theorems", Notes of the Lecture on the Prairie Analysis Seminar, DM, Kansas State University (2005).

[4] G.P. Menzala, C.F. Vasconcellos, E. Zuazua, Stabilization of the Kortweg-de Vries equation with localized damping, Quarterly of Applied Mathematics, 60, No. 1 (2002), 111-129. 
[5] L. Rosier, Exact controllability for the Korteweg-De Vries equation on a bounded domain, Control Optmization and Calculus of Variations, 2 (1997), 33-55.

[6] J.C. Saut, B. Scheurer, Unique continuation for some evolution equations, Diff. Equations, 66 (1987), 118-139.

[7] Y. Shang, Unique continuation for symmetric regularized long wave equation, Research Report of The Institute of Mathematical Sciences of The Chinese University of Hong Kong, Shatin, Hong Kong, 125 (2005).

[8] C.F. Vasconcellos, Exact controllability for the Korteweg-De Vries-Kawahara equation on a bounded domain, X ICCAM, K. U. Leuven (2002).

[9] B.-Y. Zhang, Unique continuation for the Kortweg-de Vries equation, SIAM J. Math. Anal., 23 (1991), 55-71. 